\title{
On Outage Capacity of MIMO Poisson Fading Channels
}

\author{
Kaushik Chakraborty*, Subhrakanti Dey ${ }^{\dagger}$ and Massimo Franceschetti* \\ * Department of Electrical and Computer Engineering \\ University of California at San Diego \\ La Jolla, CA 92126 \\ Email: kchakrab@ucsd.edu,massimo@ece.ucsd.edu \\ $\dagger^{\dagger}$ Department of Electrical and Electronic Engineering \\ University of Melbourne \\ Victoria 3010, Australia \\ Email: s.dey@ee.unimelb.edu.au
}

\begin{abstract}
The information outage probability of a shot-noise limited direct detection multiple-input multiple-output (MIMO) optical channel subject to block fading is considered. Information is transmitted over this channel by modulating the intensity of a number of optical signals, one corresponding to each transmit aperture, and individual photon arrivals are observed at multiple receive photodetector apertures. The transmitted signals undergo multiplicative fading, and the fading occurs in coherence intervals of fixed duration in each of which the channel fade matrix remains constant. The channel fade matrix varies across successive coherence intervals in an independent and identically distributed fashion. The transmitter and the receiver are assumed to have perfect channel state information (CSI). The main contributions are a formulation of the outage probability problem as an optimization problem and an exact characterization of the optimal solution for the special case of the MIMO Poisson fading channel with two transmit apertures.
\end{abstract}

\section{INTRODUCTION}

Free space optics is emerging as an attractive technology for several applications, e.g., metro network extensions, last mile connectivity, fiber backup, RF-wireless backhaul and enterprise connectivity [10]. The many benefits of wireless optical systems include rapid deployment time, high security, inexpensive components, seamless wireless extension of the optical fiber backbone, immunity from RF interference and lack of licensing regulations. Consequently, free space optical communication has received much attention in recent years (cf. e.g., [2], [3], [5], [6], [8], [11] and the references therein). In free space optical communication links, atmospheric turbulence causes random fluctuations in the refractive index of air at optical wavelengths, which in turn causes random fluctuations in the intensity and phase of a propagating optical signal. These intensity fluctuations, which can degrade communication performance, are typically modeled in terms of an ergodic lognormal process with a correlation time of the order of 1-10 ms. Hence, the free space optical channel can be effectively modeled as a slowly varying fading channel with occasional deep fades that can affect millions of consecutive bits [5].

Two general approaches are often followed to combat the detrimental effects of fading, viz., (a) use of estimates of the channel fade (also referred to as channel state information or $\mathrm{CSI}$ ) at the transmitter and the receiver, and (b) use of multiple transmitter and receiver elements. For radio frequency (RF) communication, comprehensive reviews of these approaches can be found in [9], [4]. In optical fading channels, instantaneous realizations of the channel state can be estimated at the receiver; then, depending on the availability of a feedback link and the amount of acceptable delay, the transmitter can be provided with complete or partial knowledge of the channel state, which can be used for adaptive power control, thereby achieving higher throughput [2], [3].

We consider a shot-noise limited direct detection MIMO optical fading channel with peak and average transmitter power constraints. At each receive aperture, the optical fields received from different transmit apertures are assumed to be sufficiently separated in frequency or angle of arrival, so that the received total power is the sum of powers from individual transmit apertures, scaled by the respective path gains [7]. We consider the same block fading channel model as proposed in [3], in which the channel fade is assumed to remain unchanged for a coherence interval of a fixed duration $T_{c}$ (seconds), and changes across successive such intervals in an independent and identically distributed (i.i.d.) fashion. A shortcoming of our model is that it ignores bandwidth limitations associated with the transmitter and receiver devices currently used in practice. We also ignore the effects of infrared and visible background light, and assume that the dark current at the photodetector is the dominant source of noise. These assumptions lead to a simple channel model which is amenable to an exact analysis. Other models have also been proposed in the literature (cf. e.g., [6], [8], [11]).

Of direct relevance to our work are the recent results of [5], [2], [3]. In [5], the authors computed upper and lower bounds on the capacity of the MIMO Poisson fading channel with perfect CSI at the transmitter and the receiver. These bounds were also used to compute approximate expressions for the capacity versus outage probability for the MIMO Poisson fading channel. In [3], an exact characterization of the capacity was obtained for the MIMO Poisson fading channel in terms of the average transmitter conditional duty cycles, conditioned on the transmitter CSI. In this paper, we extend the results of [3] to the more realistic setting of delay-limited applications, where the delay constraints may prevent coding over several coherence intervals. In this case, the capacity in the strict Shannon sense is zero, because of a nonzero probability of the channel being in such deep fade that the instantaneous mutual information is below any desired rate [9]. A more relevant performance metric is the capacity versus outage probability, 
which is a measure of the probability that the fading channel can support a desired information rate.

The remainder of the paper is organized as follows. In Section II, we provide a formal description of the outage capacity problem for the MIMO Poisson fading channel. The special case of $N=2$ transmit apertures is addressed next in Section III. In Section IV, some numerical examples are provided. Finally, Section V contains our concluding remarks.

\section{Problem Formulation}

We consider a shot-noise limited MIMO optical channel corresponding to multiple apertures at the transmitter and the receiver. We assume that the channel fades remain fixed over intervals of width $T_{c}$, and change in an i.i.d. manner across successive such intervals. For given $\mathbb{R}_{+}$-valued ${ }^{1}$ transmitted signals $\left\{x_{n}(t), t \geq 0\right\}, n=1, \ldots, N$, from the $N$ transmit apertures, the corresponding received signal at the $m^{\text {th }}$ receive aperture, $m=1, \ldots, M$, is a $\mathbb{Z}_{+}$-valued nondecreasing (leftcontinuous) Poisson counting process (PCP) $\left\{Y_{m}(t), t \geq 0\right\}$ with rate (or intensity) equal to ${ }^{2}$

$$
\Lambda_{m}(t)=\sum_{n=1}^{N} S_{n m}\left[\left\lceil t / T_{c}\right\rceil\right] x_{n}(t)+\lambda_{0 m}, t \geq 0,
$$

where $\lambda_{0 m} \geq 0$ is the (constant) dark current rate at the $m^{\text {th }}$ receive aperture, and $S_{n m}[k]$ is the $\mathbb{R}_{+}$-valued random channel fade or path gain from the $n^{\text {th }}$ transmit aperture to the $m^{\text {th }}$ receive aperture in the $k^{\text {th }}$ coherence interval. We shall assume throughout that the transmitter and the receiver have perfect CSI.

With $[0, T]$ being the time interval of transmission and reception over the channel, the channel input from the $n^{t h}$ aperture is a $\mathbb{R}_{+}$-valued signal $\left\{x_{n}(t), 0 \leq t \leq T\right\}$, which is proportional to the transmitted optical power, and which satisfies peak power constraints and an average sum power constraint of the form

$$
\begin{aligned}
0 \leq x_{n}(t) & \leq A_{n}, \quad 0 \leq t \leq T, \\
\frac{1}{T} \int_{0}^{T} \sum_{n=1}^{N} x_{n}(t) d t & \leq \sigma \sum_{n=1}^{N} A_{n},
\end{aligned}
$$

where $A_{n}>0, n=1, \ldots, N$, and $0 \leq \sigma \leq 1$ are fixed. Here $A_{n}$ specifies the maximum instantaneous value of the intensity of the optical signal transmitted from the $n^{t h}$ transmit aperture, $n=1, \ldots, N$, and $\sigma$ specifies the weighted sum of the ratio of the average-to-peak power from all the transmitted apertures.

In [3], it was established that the optimal transmission scheme that achieves channel capacity is binary signaling through each transmit aperture with arbitrarily fast intertransition times. The two signaling levels correspond to no transmission ("OFF" state) and transmission at the peak power level ("ON" state). The conditional probability that the $n^{\text {th }}$ transmit aperture is in the ON state when the channel state is $\mathbf{s} \in \mathbb{R}_{+}^{N M}$ can be seen as the average conditional "duty cycle" of the $n^{t h}$ transmit aperture, $n=1, \ldots, N$. In general, the transmitted signals are correlated across apertures but are i.i.d. in time. Furthermore, whenever a transmit aperture is in the ON state, then all the transmit apertures with the same or higher values of average conditional duty cycles must

\footnotetext{
${ }^{1}$ We denote the set of nonnegative real numbers by $\mathbb{R}_{+}$and the set of nonnegative integers by $\mathbb{Z}_{+}$.

${ }^{2}$ The notation $\lceil x\rceil$ denotes the smallest integer greater than or equal to $x$.
}

also remain ON. Using these facts, the instantaneous mutual information for the $N \times M$ Poisson fading channel, when the instantaneous channel fade is $\mathbf{s}=\left\{s_{n m}\right\}_{n=1, m=1}^{N, M} \in \mathbb{R}_{+}^{N \times M}$ and the average conditional duty cycles are $\boldsymbol{\mu}^{N}=\left\{\mu_{n}, n=\right.$ $1, \ldots, N\} \in[0,1]^{N}$, is obtained as [3]

$$
\begin{aligned}
& I\left(\boldsymbol{\mu}^{N}, \mathbf{s}\right) \\
& =\sum_{m=1}^{M}\left[\sum_{n=1}^{N} \nu_{n} \zeta\left(\sum_{k=1}^{n} s_{\Pi(k) m} A_{\Pi(k)}, \lambda_{0 m}\right)\right. \\
& \left.\quad-\zeta\left(\sum_{n=1}^{N} \nu_{n} \sum_{k=1}^{n} s_{\Pi(k) m} A_{\Pi(k)}, \lambda_{0 m}\right)\right],
\end{aligned}
$$

where we have defined

$$
\zeta(x, y) \triangleq(x+y) \log (x+y)-y \log y, \quad x, y \geq 0
$$

(with the convention $0 \log 0 \triangleq 0$ ); $\Pi:\{1, \cdots, N\} \rightarrow$ $\{1, \cdots, N\}$ is a permutation of $\{1, \cdots, N\}$ such that

$$
\mu_{\Pi(n)} \geq \mu_{\Pi(n+1)}, n=1, \cdots, N-1,
$$

and

$$
\nu_{n} \triangleq \begin{cases}\mu_{\Pi(n)}-\mu_{\Pi(n+1)}, & n=1, \cdots, N-1, \\ \mu_{\Pi(N)}, & n=N .\end{cases}
$$

Noting that $\zeta(., y)$ is strictly convex on $[0, \infty)$ for every $y \geq$ 0 , it can be verified that the instantaneous mutual information is a strictly concave function of the duty cycles $\left\{\mu_{n}\right\}_{n=1}^{N}$ [3]. It can be also shown that for every $\mathbf{s} \in \mathbb{R}_{+}^{N M}, I\left(\boldsymbol{\mu}^{N}, \mathbf{s}\right)$ is continuous but not differentiable along the planes $\mu_{i}=\mu_{j}$, $i, j \in\{1, \ldots, N\}, i \neq j$.

We are now ready to introduce the outage capacity optimization problem for the $N \times M$ MIMO Poisson fading channel under average peak power constraints $\left\{A_{n}\right\}_{n=1}^{N}$, and an average sum power constraint $\sigma$ :

Problem P: Given a basic rate $r_{0} \geq 0$, minimize $P\left(I\left(\boldsymbol{\mu}^{N}(\mathbf{S}), \mathbf{S}\right)<r_{0}\right)$ subject to $0 \leq \mu_{n}(\mathbf{S}) \leq 1$ with probability 1 and $\mathbb{E}\left[\sum_{n=1}^{N} \frac{A_{n}}{\sum_{n=1}^{N} A_{n}} \mu_{n}(\mathbf{S})\right] \leq \sigma$.

The solution to the optimization problem $\mathbf{P}$ will be referred to as the information outage probability of the MIMO Poisson fading channel evaluated at rate $r_{0}$ subject to the aforementioned peak and average sum power constraints. One major difficulty in obtaining a closed form analytical solution for the outage probability is the nondifferentiability of $I(\cdot, \mathbf{s})$; standard variational techniques for differentiable functions cannot be directly applied here. Nonsmooth optimization techniques can be applied to determine the solution computationally, but this is beyond the scope of this paper that seeks an analytic solution. Our approach is to partition the $N$-dimensional unit hypercube spanning the feasible range of average conditional duty cycles $\boldsymbol{\mu}^{N}$ into subsets in which the instantaneous mutual information is smooth, and apply standard optimization tools, e.g., Karush-Kuhn-Tucker (KKT) conditions, in these subsets. The boundaries of these subsets, in which the instantaneous mutual information is nondifferentiable, is treated separately. Although in theory, this approach can lead to a characterization of the outage probability problem $\mathbf{P}$ for an arbitrary positive integer $N$, the exact characterization is extremely cumbersome, and we limit our treatment to the special case of $N=2$ transmit apertures. 


\section{Outage Capacity With $N=2$ Transmit APERTURES}

In this section, we study the outage capacity problem for a $2 \times M$ MIMO Poisson fading channel, which corresponds to a wireless optical communication system with 2 transmit apertures and $M$ receive apertures. In stating our results, it is convenient to set

$$
\begin{aligned}
& \xi(x)=\frac{1}{x}\left(e^{-1}(1+x)^{(1+1 / x)}-1\right), \quad x \geq 0, \\
& h(x)=(1+x) \log (1+x)-x, \quad x \geq 0 .
\end{aligned}
$$

We also use the notation $b_{n m}=\frac{s_{n m} A_{i}}{\lambda_{0 m}}, n=1,2, B_{m}=b_{1 m}+$ $b_{2 m}, m=1, \ldots, M, \mathbf{b} \triangleq\left(b_{11}, \ldots, b_{1 M}, b_{21}, \ldots, b_{2 M}\right) \in$ $\mathbb{R}_{+}^{2 M}$, and $a \triangleq \frac{A_{1}}{A_{1}+A_{2}}$.

For $N=2$ transmit apertures and $M \geq 1$ receive apertures, the instantaneous mutual information can be written as (via some algebraic manipulations of (3))

$$
\begin{aligned}
& I\left(\mu_{1}, \mu_{2}, \mathbf{s}\right) \\
& = \begin{cases}\sum_{m=1}^{M} \lambda_{0 m}\left\{\left(\mu_{1}-\mu_{2}\right) h\left(b_{1 m}\right)+\mu_{2} h\left(B_{m}\right)\right. \\
\left.-h\left(\mu_{1} b_{1 m}+\mu_{2} b_{2 m}\right)\right\}, & \text { if } \mu_{1} \geq \mu_{2}, \\
\sum_{m=1}^{M} \lambda_{0 m}\left\{\left(\mu_{2}-\mu_{1}\right) h\left(b_{2 m}\right)+\mu_{1} h\left(B_{m}\right)\right. & \text { if } \mu_{1}<\mu_{2}, \\
\left.-h\left(\mu_{1} b_{1 m}+\mu_{2} b_{2 m}\right)\right\}, & \text { r }\end{cases}
\end{aligned}
$$

where we have suppressed the dependence of the average conditional duty cycles $0 \leq \mu_{1}, \mu_{2} \leq 1$ on the channel fade for notational convenience.

The outage capacity optimization problem for the $2 \times M$ MIMO Poisson fading channel subject to peak power constraints $A_{1}, A_{2}$ and an average sum power constraint $\sigma$, is given by:

Problem P1: Given a basic rate $r_{0} \geq 0$, minimize $P\left(I\left(\mu_{1}, \mu_{2}, \mathbf{S}\right)<r_{0}\right)$ subject to $0 \leq \mu_{1}, \mu_{2} \leq 1$ and $\mathbb{E}\left[a \mu_{1}+(1-a) \mu_{2}\right] \leq \sigma$.

In order to solve the above problem, we need to first solve the following problem:

Problem P1a: For a given channel realization $\mathbf{s} \in \mathbb{R}_{+}^{2 M}$, minimize $\left(a \mu_{1}+(1-a) \mu_{2}\right)$ subject to $0 \leq \mu_{1}, \mu_{2} \leq 1$ and $I\left(\mu_{1}, \mu_{2}, \mathbf{s}\right) \geq r_{0}$

Once the optimal solution to P1a is obtained, one can characterize the solution to $\mathbf{P 1}$ in terms of the solution to P1a and the average sum power constraint. For similar techniques in outage capacity optimization for block-fading AWGN channels, see [1].

Note that at optimality for P1a, we must have $I\left(\mu_{1}, \mu_{2}, \mathbf{s}\right)=r_{0}$ as otherwise one can lower any of $\mu_{1}, \mu_{2}$ to achieve equality and in the process improve (lower) the value of the objective function. However, unlike the Shannon capacity for the AWGN channel, the instantaneous mutual information for the Poisson fading channel is not monotonically increasing, although it is concave (for the SISO case, see [2]). This implies that for a given channel realization, the mutual information $I\left(\mu_{1}, \mu_{2}, \mathbf{s}\right)$ achieves a maximum for some $\mu_{1}, \mu_{2}$ and for feasibility, we need to verify whether the basic rate $r_{0}$ is less than or equal to this maximum mutual information for feasibility, given a particular channel realization. Thus we need to first solve the following optimization problem:

Problem P2: Given a particular channel realization $\mathbf{s} \in \mathbb{R}_{+}^{2 M}$ in a given fading block, maximize $I\left(\mu_{1}, \mu_{2}, \mathbf{s}\right)$ subject to $0 \leq$ $\mu_{1}, \mu_{2} \leq 1$
Lemma 3.1: The optimal solution to Problem $\mathbf{P 2}$ is given by $\mu_{1}=\mu_{2}=\mu_{\text {opt }}$ where $\mu_{\text {opt }}$ satisfies

$$
\sum_{m=1}^{M} \lambda_{0 m} B_{m} \log \left(\frac{1+B_{m} \xi\left(B_{m}\right)}{1+\mu_{o p t} B_{m}}\right)=0,
$$

and the maximum mutual information is given by

$$
r_{\max }(\mathbf{s})=\sum_{m=1}^{M} \lambda_{0 m}\left[\mu_{o p t} B_{m}-\log \left(1+\mu_{o p t} B_{m}\right)\right] .
$$

The proof of Lemma 3.1 is omitted due to space constraints. Notice that this lemma implies that regardless of any average sum power constraint, a basic rate $r_{0}$ may not be feasible for a particular channel realization $\mathbf{s} \in \mathbb{R}_{+}^{2 M}$ if $r_{0}>r_{\max }(\mathbf{s})$, and an outage will occur. In this case the optimal duty cycles are clearly $\mu_{1}^{*}=\mu_{2}^{*}=0$. When $r_{0}=r_{\max }(\mathbf{s})$, the maximum achievable instantaneous mutual information, clearly, the optimal duty cycles are $\mu_{1}^{*}=\mu_{2}^{*}=\mu_{\text {opt }}$. In what follows, we will denote feasibility for a given channel state by assuming $r_{0}$ is strictly less than the maximum value given by Lemma 3.1.

The following Lemma presents the solution to Problem P1a where the optimal duty cycles are denoted as $\mu_{1}^{*}, \mu_{2}^{*}$. Once again, we omit the proof due to space constraints.

Lemma 3.2: Given a basic rate $r_{0} \geq 0$ and a particular channel realization (for a given fading block) $\mathbf{s}$, if $r_{0}>$ $r_{\max }(\mathbf{s})$, the optimal duty cycles are clearly $\mu_{1}^{*}=\mu_{2}^{*}=0$. When $r_{0}=r_{\max }(\mathbf{s})$, the optimal duty cycles are $\mu_{1}^{*}=\mu_{2}^{*}=$ $\mu_{\text {opt }}$. When $r_{0}<r_{\max }(\mathbf{s}), \mu_{1}^{*}, \mu_{2}^{*}$ are given by one of the following five cases:

Case 1: Suppose there exist $\mu^{*}, \lambda_{1}^{*}>0, \rho_{1}^{*}, \rho_{2}^{*} \geq 0$ such that

$$
\mu^{*}=\frac{r_{0}+\sum_{m=1}^{M} \lambda_{0 m} h\left(\mu^{*} B_{m}\right)}{\sum_{m=1}^{M} \lambda_{0 m} h\left(B_{m}\right)},
$$

$$
\begin{aligned}
& \sum_{m=1}^{M} \lambda_{0 m}\left\{h\left(b_{1 m}\right)-b_{1 m} \log \left(1+\mu^{*} B_{m}\right)\right\}=\frac{a-\rho_{1}^{*}}{\lambda_{1}^{*}}, \\
& \sum_{m=1}^{M} \lambda_{0 m}\left\{h\left(b_{2 m}\right)-b_{2 m} \log \left(1+\mu^{*} B_{m}\right)\right\}=\frac{1-a-\rho_{2}^{*}}{\lambda_{1}^{*}}, \\
& \sum_{m=1}^{M} \lambda_{0 m}\left\{h\left(B_{m}\right)-B_{m} \log \left(1+\mu^{*} B_{m}\right)\right\}=\frac{1}{\lambda_{1}^{*}} .
\end{aligned}
$$

Then $\mu_{1}^{*}=\mu_{2}^{*}=\mu^{*}>0$.

Case 2: Suppose there exist $\bar{\mu}_{1}>\bar{\mu}_{2}>0, \bar{\lambda}_{1}^{*}>0$ such that

$$
\begin{gathered}
\sum_{m=1}^{M} \lambda_{0 m}\left\{\bar{\mu}_{1} h\left(b_{1 m}\right)+\bar{\mu}_{2}\left(h\left(B_{m}\right)-h\left(b_{1 m}\right)\right)\right\} \\
=r_{0}+\sum_{m=1}^{M} \lambda_{0 m}\left(h\left(\bar{\mu}_{1} b_{1 m}+\bar{\mu}_{2} b_{2 m}\right)\right), \\
\sum_{m=1}^{M} \lambda_{0 m}\left\{h\left(b_{1 m}\right)-b_{1 m} \log \left(1+\bar{\mu}_{1} b_{1 m}+\bar{\mu}_{2} b_{2 m}\right)\right\}=\frac{a}{\lambda_{1}^{*}}, \\
\sum_{m=1}^{M} \lambda_{0 m}\left\{h\left(B_{m}\right)-B_{m} \log \left(1+\bar{\mu}_{1} b_{1 m}+\bar{\mu}_{2} b_{2 m}\right)\right\}=\frac{1}{\lambda_{1}^{*}} .
\end{gathered}
$$

Then $\mu_{1}^{*}=\bar{\mu}_{1}, \mu_{2}^{*}=\bar{\mu}_{2}$.

Case 3: Suppose there exist $\tilde{\mu}_{1}>0, \tilde{\lambda}_{1}^{*}>0$ such that

$$
\begin{gathered}
\tilde{\mu}_{1}=\frac{r_{0}+\sum_{m=1}^{M} \lambda_{0 m} h\left(\tilde{\mu}_{1} b_{1 m}\right)}{\sum_{m=1}^{M} \lambda_{0 m} h\left(b_{1 m}\right)}, \\
\sum_{m=1}^{M} \lambda_{0 m}\left\{h\left(b_{1 m}\right)-b_{1 m} \log \left(1+\tilde{\mu}_{1} b_{1 m}\right)\right\}=\frac{a}{\tilde{\lambda}_{1}^{*}}, \\
\sum_{m=1}^{M} \lambda_{0 m}\left\{h\left(B_{m}\right)-B_{m} \log \left(1+\tilde{\mu}_{1} b_{1 m}\right)\right\} \leq \frac{1}{\tilde{\lambda}_{1}^{*}} .
\end{gathered}
$$

Then $\mu_{1}^{*}=\tilde{\mu}_{1}, \mu_{2}^{*}=0$.

Case 4: Suppose there exist $\hat{\mu}_{2}>\hat{\mu}_{1}>0, \lambda_{2}^{*}>0$ such that

$$
\begin{gathered}
\sum_{m=1}^{M} \lambda_{0 m}\left\{\hat{\mu}_{1}\left(h\left(B_{m}\right)-h\left(b_{2 m}\right)\right)+\hat{\mu}_{2} h\left(b_{2 m}\right)\right\} \\
=\quad r_{0}+\sum_{m=1}^{M} \lambda_{0 m} h\left(\hat{\mu}_{1} b_{1 m}+\hat{\mu}_{2} b_{2 m}\right),
\end{gathered}
$$




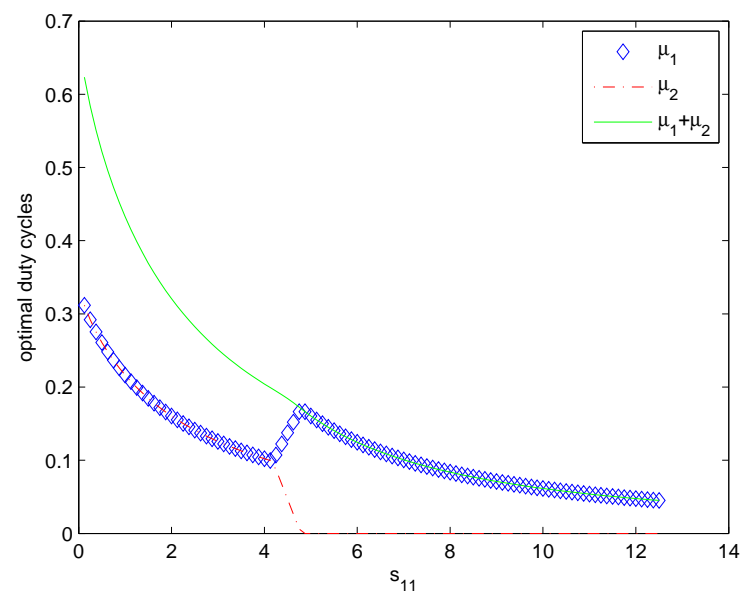

Fig. 1. Plot of the optimal duty cycles versus increasing $s_{11}$.

$\sum_{m=1}^{M} \lambda_{0 m}\left\{h\left(b_{2 m}\right)-b_{2 m} \log \left(1+\hat{\mu}_{1} b_{1 m}+\hat{\mu}_{2} b_{2 m}\right)\right\}=\frac{1-a}{\lambda_{2}^{*}}$
$\sum_{m=1}^{M} \lambda_{0 m}\left\{h\left(B_{m}\right)-B_{m} \log \left(1+\hat{\mu}_{1} b_{1 m}+\hat{\mu}_{2} b_{2 m}\right)\right\}=\frac{1}{\lambda_{2}^{*}}$.

Then $\mu_{1}^{*}=\hat{\mu}_{1}, \mu_{2}^{*}=\hat{\mu}_{2}$.

Case 5: Suppose there exist $\mu_{2}^{\prime}>0, \tilde{\lambda}_{2}^{*}>0$ such that

$$
\begin{gathered}
\mu_{2}^{\prime}=\frac{r_{0}+\sum_{m=1}^{M} \lambda_{0 m} h\left(\mu_{2}^{\prime} b_{2 m}\right)}{\sum_{m=1}^{M} \lambda_{0 m} h\left(b_{2 m}\right)}, \\
\sum_{m=1}^{M} \lambda_{0 m}\left\{h\left(b_{2 m}\right)-b_{2 m} \log \left(1+\mu_{2}^{\prime} b_{2 m}\right)\right\}=\frac{1-a}{\tilde{\lambda}_{2}^{*}}, \\
\sum_{m=1}^{M} \lambda_{0 m}\left\{h\left(B_{m}\right)-B_{m} \log \left(1+\mu_{2}^{\prime} b_{2 m}\right)\right\} \leq \frac{1}{\tilde{\lambda}_{2}^{*}} .
\end{gathered}
$$

Then $\mu_{1}^{*}=0, \mu_{2}^{*}=\mu_{2}^{\prime}$.

We now present the following result, again without proof:

Lemma 3.3: Given $r_{0}$ is feasible, the optimal sum duty cycle for a given channel realization given by $a \mu_{1}^{*}+(1-a) \mu_{2}^{*}$, is a continuous and nonincreasing function of any of the channel gains $s_{i j}, i=1,2, j=1, \ldots, M$.

Figure 1 shows how the optimal conditional duty cycles vary for a $2 \times 2$ MIMO Poisson fading channel, with increasing $s_{11}$ while $s_{12}, s_{21}$ and $s_{22}$ are kept fixed. Here $\lambda_{01}=\lambda_{02}=1$, $A_{1}=A_{2}=1$, so that $a=\frac{1}{2}$, and the target instantaneous mutual information is $r_{0}=1$ nats/secs/ $\mathrm{Hz}$. The various regions $\mu_{1}^{*}=\mu_{2}^{*}=0$ (within the outage set), $\mu_{1}^{*}=\mu_{2}^{*}>0, \mu_{1}^{*}>\mu_{2}^{*}>$ 0 and $\mu_{1}^{*}>\mu_{2}^{*}=0$ can be easily seen while the optimal sum duty cycle decreases with increasing $s_{11}$ within the feasible set.

With the two Lemmas 3.2 and 3.3 established, we can now present the complete solution to Problem P1. We introduce the following definitions:

$$
\begin{aligned}
& \mathcal{R}(p)=\left\{\mathbf{b} \in \mathbb{R}_{+}^{2 M}:\langle\mu\rangle<p\right\}, \\
& \overline{\mathcal{R}}(p)=\left\{\mathbf{b} \in \mathbb{R}_{+}^{2 M}:\langle\mu\rangle \leq p\right\},
\end{aligned}
$$

where for convenience we have used the notation $\langle\mu\rangle=\left[a \mu_{1}+\right.$ $\left.(1-a) \mu_{2}\right]$. The boundary surface $\mathcal{B}(p)$ of $\mathcal{R}(p)$ is defined as the set of points $\mathbf{b}$ such that $\langle\mu\rangle=p$. We further define the following two average duty cycle sums as

$$
\Sigma(p)=\int_{\mathcal{R}(p)}\langle\mu\rangle d F(\mathbf{b}), \bar{\Sigma}(p)=\int_{\overline{\mathcal{R}}(p)}\langle\mu\rangle d F(\mathbf{b}),
$$

where $F(\mathbf{b})$ is the c.d.f. of $\mathbf{b}$. The duty cycle sum threshold $p^{*}$ is defined as $p^{*}=\sup \{p: \Sigma(p)<\sigma\}$ and the weight $w^{*}$ is defined as

$$
w^{*}=\frac{\sigma-\Sigma\left(p^{*}\right)}{\bar{\Sigma}\left(p^{*}\right)-\Sigma\left(p^{*}\right)} .
$$

The following theorem, which summarizes the solution to Problem P1, constitutes the main result of this paper. The proof follows the techniques of Proposition 4 of [1] (see Appendix $\mathrm{D}$ in [1]).

Theorem 1: If $\mathbb{E}\left[a \mu_{1}^{*}+(1-a) \mu_{2}^{*}\right] \leq \sigma$, where $\mu_{1}^{*}, \mu_{2}^{*}$ are given by Lemma 3.2, the optimal duty cycles that solve Problem P1 are given by $\mu_{1}=\mu_{1}^{*}, \mu_{2}=\mu_{2}^{*}$. On the other hand, if $\mathbb{E}\left[a \mu_{1}^{*}+(1-a) \mu_{2}^{*}\right]>\sigma$, the solution to $\mathbf{P 1}$ is given by:

$$
\begin{array}{cccc}
\mu_{1}=\mu_{1}^{*}, & \mu_{2}=\mu_{2}^{*}, & \text { if } & \mathbf{b} \in \mathcal{R}\left(p^{*}\right), \\
\mu_{1}=0, & \mu_{2}=0, & \text { if } & \mathbf{b} \notin \overline{\mathcal{R}}\left(p^{*}\right) .
\end{array}
$$

If $\mathbf{b} \in \mathcal{B}\left(p^{*}\right)$, then $\mu_{1}=\mu_{1}^{*}, \mu_{2}=\mu_{2}^{*}$ with probability $w^{*}$ and $\mu_{1}=0, \mu_{2}=0$ with probability $1-w^{*}$.

Remark: The above result implies that when the average sum duty cycle constraint is active, that is, $\mathbb{E}\left[a \mu_{1}^{*}+(1-a) \mu_{2}^{*}\right]>$ $\sigma$, the optimal duty cycle allocation amounts to finding an optimal threshold $p^{*}$ such that when $a \mu_{1}^{*}+(1-a) \mu_{2}^{*}>p^{*}$, the transmitters are turned off and they are turned on when $a \mu_{1}^{*}+(1-a) \mu_{2}^{*}<p^{*}$, where $\mu_{1}^{*}, \mu_{2}^{*}$ are the solutions to Problem P1a, i.e, they achieve the minimum sum duty cycle while meeting the basic rate $r_{0}$. The threshold $p^{*}$ is chosen such that the average (long-term) sum duty cycle constraint is equal to $\sigma$. If $F(\mathbf{b})$ is not continuous, then a randomization is necessary when $a \mu_{1}^{*}+(1-a) \mu_{2}^{*}=p^{*}$, and $w^{*}$, the probability of transmitting is chosen to satisfy the long term sum duty cycle constraint $\mathbb{E}\left[a \mu_{1}+(1-a) \mu_{2}\right]=\sigma$. Although, in the case of the free-space optical fading channel, usually the fading distribution is believed to be continuous (log-normal) and thus, the value of $w^{*}$ can be chosen to be any real number between 0 and 1 without affecting optimality.

The outage probability can be obtained as

$1-\int_{\overline{\mathcal{R}}\left(p^{*}\right)} d F(\mathbf{b})=1-w^{*} P\left(\mathbf{b} \in \mathcal{B}\left(p^{*}\right)\right)-P\left(\mathbf{b} \in \mathcal{R}\left(p^{*}\right)\right)$.

\section{NUMERICAL STUDIES}

In this section, we present some illustrative simulation results for a $2 \times 2$ MIMO Poisson fading channel where $S_{11}$, $S_{12}, S_{21}$ and $S_{22}$ are i.i.d. lognormal random variables such that $\frac{1}{2} \log S_{i j} \sim \mathcal{N}\left(\mu_{G}, \sigma_{G}^{2}\right)$. As in [2], we take $\mu_{G}=-\sigma_{G}^{2}$ such that the fade is normalized i.e, $E\left[S_{i j}\right]=1$. We consider $\sigma_{G}^{2}=0.1$, which corresponds to a moderately turbulent fade. We also take (for simplicity) $A_{1}=A_{2}=1$ (or $a=0.5)$ and $\lambda_{01}=\lambda_{02}=1$. For the simulations, we choose $r_{0}=0.25$ nats/ unit time, whereas the expected unconstrained optimal mutual information, i.e., $\mathbb{E}\left[r_{\max }(\mathbf{S})\right]$ was found to be approximately 0.57 nats/unit time. The following plots are obtained through computer simulations averaged over 100,000 channel realizations.

Figure 2 illustrates how $\Sigma\left(p^{*}\right)$ varies with $p^{*}$, the optimal sum duty cycle threshold. In practice, one can use this graph to obtain $p^{*}$ for a given average sum duty cycle threshold constraint $\sigma$. It was noticed that the unconstrained optimal average sum duty cycle $\mathbb{E}\left[\frac{1}{2}\left(\mu_{1}^{*}+\mu_{2}^{*}\right)\right]=\sigma_{0}=0.12$ (approximately). So, for all $\sigma$ larger than this value, the optimal duty cycle allocation law is given by Lemma 3.2. Note also that 


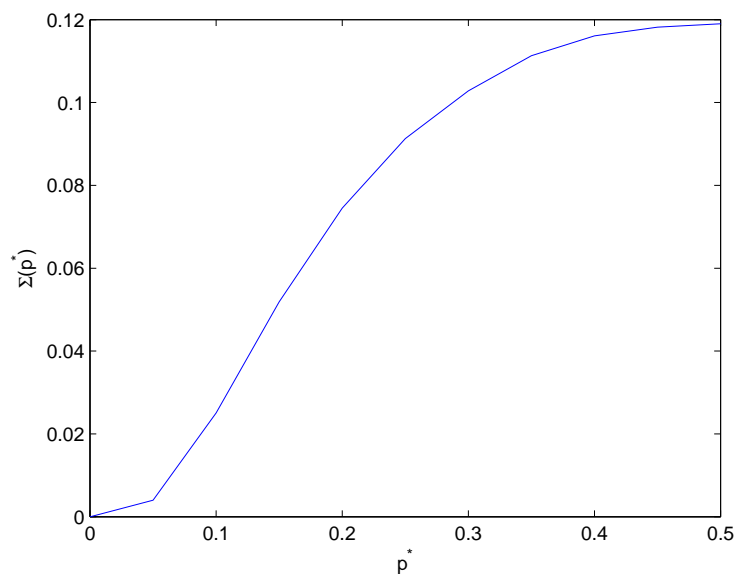

Fig. 2. Plot of average sum duty cycle versus threshold $p^{*}$.

the choice of $w^{*}$ is not crucial here as the fade distribution is continuous and hence $w^{*}$ can be chosen as any real number in $[0,1]$. We chose $w^{*}=0.5$. Figure 3 shows the outage performance of the optimal duty cycle allocation algorithm as opposed to a constant duty cycle allocation $\mu_{1}=\mu_{2}=\sigma$ (such that the average sum duty cycle constraint is satisfied). It is easily seen that the optimal allocation scheme outperforms the constant duty cycle allocation scheme quite substantially. As $\sigma \rightarrow \sigma_{0}$, the outage probability clearly attains a floor (as expected), since for all $\sigma>\sigma_{0}$, the outage probability is given by the probability that the maximum achievable mutual information (for a given channel fade) falls below the basic rate $r_{0}$. Thus, increasing average power does not reduce outage probability beyond this point. Recall that this is due to the fact that for optical wireless transmission over Poisson fading channels, the instantaneous mutual information is a concave but not a monotonically increasing function of the duty cycles, as opposed to the AWGN fading channel. Note also that if one further lowered the basic rate requirement $r_{0}$, the unconstrained average sum duty cycle $\sigma_{0}$ will increase beyond 0.12 and gradually approach 0.5 . However, due to space limitations we do not provide further graphs as they are similar to the ones provided here.

We do not provide a direct comparison of these results with the existing upper and lower bounds on the outage capacity derived in [5] due to the following reasons. In [5], individual average power constraints were imposed on all the transmit apertures, while here we consider a constraint on the sum of the average powers across all transmit apertures. Therefore, the optimal allocation of duty cycles as computed in Theorem 1 do not constitute the optimal solution for the problem considered in [5]. We remark that we provided an exact solution to the outage capacity problem under our formulation and that it is also possible to use the techniques outlined in this paper to solve the outage capacity problem with individual average power constraints, but we do not discuss it in this paper.

\section{DISCUSSION}

We have studied the outage capacity problem for a singleuser shot-noise limited direct detection block fading MIMO Poisson channel. Under the assumption of perfect transmitter and receiver CSI, a characterization of the information outage

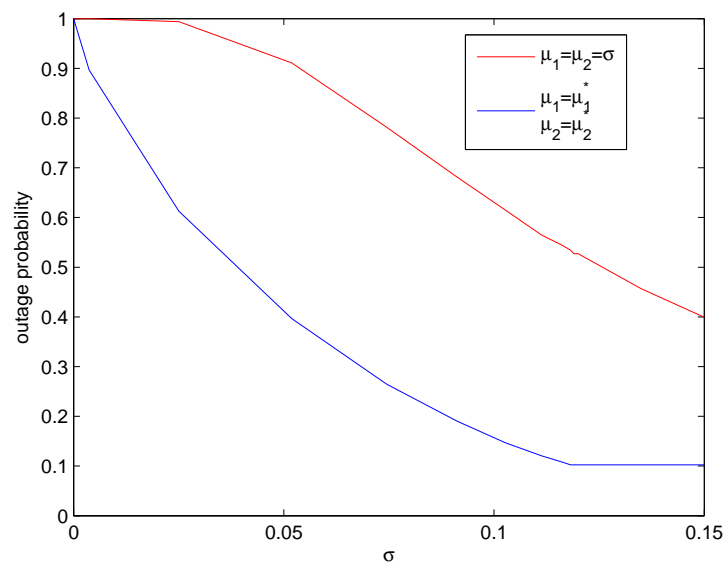

Fig. 3. Plot of outage probability versus average sum power constraint $\sigma$.

probability is obtained when the transmitted signals from the different transmit apertures are subject to peak and average sum power constraints. For the special case of two transmit apertures, the optimal average conditional duty cycles, and hence the outage capacity, have been explicitly determined.

The exact value of the average sum power constraint $\sigma$ plays a critical role in the characterization of the optimal duty cycles. There are two distinct regimes, depending on whether the average sum power constraint is active or inactive. However, regardless of the value of $\sigma$, a basic rate $r_{0}$ will not be feasible for channel states s such that $r_{0}>r_{\max }(\mathbf{s})$, where $r_{\max }(\mathbf{s})$ satisfies (10), and denotes the maximum supportable instantaneous mutual information for the channel state $\mathbf{s}$. These channel states will always be in the outage set for the basic rate $r_{0}$, and cumulatively constitute the floor of the outage probability performance.

\section{REFERENCES}

[1] G. Caire, G. Taricco and E. Biglieri, "Optimum power control over fading channels," IEEE Trans. Inform. Theory, vol. 45, no. 5, pp. 1468-1489, July 1999.

[2] K. Chakraborty and P. Narayan, "Capacity of the SISO Poisson fading channel," IEEE Trans. Inform. Theory, revised version under review, Dec. 2006.

[3] K. Chakraborty, "Capacity of the MIMO optical fading channel," Proc. Intl. Symposium Inform. Theory 2005, pp. 530-534, Sept. 2005, Adelaide, Australia.

[4] A. Goldsmith, S. A. Jafar, N. Jindal and S. Vishwanath, "Capacity limits of MIMO channels," IEEE J. Select. Areas Commun., vol. 21, no. 5, pp. 684-702, June 2003.

[5] S. M. Haas and J. H. Shapiro, "Capacity of wireless optical communications," IEEE J. Select. Areas Commun., vol. 21, no. 8, pp. 1346-1357, Oct. 2003.

[6] S. Hranilovic and F. R. Kschischang, "Capacity bounds for power- and band-limited optical intensity channels corrupted by Gaussian noise,' IEEE Trans. Inform. Theory, vol. 50, no. 5, pp. 784-795, May 2004.

[7] A. Lapidoth and S. Shamai, "The Poisson multiple-access channel," IEEE Trans. Inform. Theory, vol. 44, no. (2), pp. 488-501, Mar. 1998.

[8] E. J. Lee and V. W. S. Chan, "Part 1: Optical communication over the clear turbulent atmospheric channel using diversity," IEEE J. Select. Areas Commun., vol. 22, no. 9, pp. 1896-1906, Nov. 2004.

[9] J. Proakis, E. Biglieri and S. Shamai, "Fading channels: informationtheoretic and communications aspects," IEEE Trans. Inform. Theory, vol. 44, no. 6, pp. 2619-2692, Oct. 1998.

[10] H. A. Willebrand and B. S. Ghuman, "Fiber optics without fiber," IEEE Spectr., vol. 38, no. 8, pp. 41-45, Aug. 2001

[11] X. Zhu and J. M. Kahn, "Free-space optical communication through atmospheric turbulent channels," IEEE Trans. Commun., vol. 50, no. 8, pp. 1293-1300, Aug. 2002. 